\title{
TRATAMENTO CIRÚRGICO DO AMELOBLASTOMA COM RECONSTRUÇÃO DE MANDÍBULA COM ENXERTO DE CRISTA ILÍACA NÃO VASCULARIZADO - ESTUDO DE SETE CASOS
}

\author{
SURGICAL TREATMENT OF THE AMELOBLASTOMA OF THE MANDIBLE AND \\ RECONSTRUCTION WITH NON VASCULARIZED ILIAC CREST GRAFT - \\ STUDY OF SEVEN CASES
}

\author{
Daniela Venturoli Lunardi ${ }^{1}$ \\ Antonio Sérgio Fava ${ }^{2}$ \\ Rui Henriques Martins ${ }^{1}$ \\ Maria da Graça Naclério Homem ${ }^{3}$ \\ Abrão Rapoport, TCBC-SP ${ }^{4}$ \\ Marcos Brasilino de Carvalho
}

\begin{abstract}
RESUMO: Objetivo: Determinar a validade do enxerto autólogo de crista ilíaca não vascularizado no tratamento cirúrgico do ameloblastoma de mandíbula. Método: Nos Serviços de Cirurgia de Cabeça e Pescoço e Semiologia Bucal do Complexo Hospitalar Heliópolis, de 1980 a 2000, foram tratados 31 pacientes com ameloblastoma de mandíbula, dos quais sete receberam enxerto de crista ilíaca autólogo, fixos com placa de titânio do sistema A0 (quatro casos) e aço inox (três casos), sendo portadores da variedade folicular (seis casos) e plexiforme (um caso). Resultados: Nesta análise, foi utilizado o Teste de Hipótese para a média populacional com a variança desconhecida, houve exposição da placa em três casos (40\%) quando a neoplasia ultrapassava a linha média e em quatro casos (60\%) não incidiu nenhuma complicação. Conclusões: Apesar da incidência de exposição de placa, o método é indicado na reconstrução da mandíbula de pacientes com ameloblastoma.
\end{abstract}

Descritores: Ameloblastoma; Crista ilíaca; Reconstrução; Placas.

\section{INTRODUÇÃO}

O termo ameloblastoma foi utilizado pela primeira vez $^{1}$ em 1930 quando foi descrito um tumor odontogênico com formação de múltiplos cordões e lâminas celulares interconectadas, de origem epitelial e homólogas com a lâmina dentogengival do início da odontogênese. A classificação desta neoplasia pela WHO (Word Health Organi- zation) situa como tumor derivado do epitélio odontogênico sem ectomesênquima ${ }^{2}$. A despeito das tentativas de uniformização da sua classificação, historicamente a subdivisão em folicular, plexiforme e cístico é a que prevalece ${ }^{3}$, apesar da existência da variedade desmoplásica ${ }^{4}$, sendo todas de localização predominantemente mandibular ${ }^{5,6}$.

Quanto ao tratamento cirúrgico destas neoplasias, este é norteado pelo tipo clínico (sólido ou cístico) e pela varie-

1. Mestre em Cirurgia de Cabeça e Pescoço do Complexo Hospitalar Heliópolis.

2. Cirurgião do Serviço de Cirurgia de Cabeça e Pescoço do Complexo Hospitalar Heliópolis.

3. Chefe do Serviço de Semiologia Bucal do Complexo Hospitalar Heliópolis e Docente Livre em Cirurgia Buco-MaxiloFacial pela Faculdade de Odontologia da USP.

4. Coordenador do Curso de Pós-Graduação em Cirurgia de Cabeça e Pescoço do Complexo Hospitalar Heliópolis e Docente Livre em Cirurgia de Cabeça e Pescoço pela Faculdade de Medicina da USP.

5. Chefe do Serviço de Cirurgia de Cabeça e Pescoço do Complexo Hospitalar Heliópolis, Doutor em Cirurgia pela Faculdade de Ciências Médicas da UNICAMP.

Recebido em 26/7/2000

Aceito para publicação em 11/10/2000

Trabalho realizado nos Serviços de Cirurgia de Cabeça e Pescoço e Semiologia Bucal do Complexo Hospitalar Heliópolis, Hosphel - São Paulo-SP. 
dade histológica, sendo conservador (enucleação ou curetagem $)^{7}$ ou não (ressecção marginal ou segmentar), esta última através de enxerto ósseo autógeno de crista ilíaca ou fíbula ${ }^{8}$, vascularizado ou não ${ }^{9}$. Para a estabilização do enxerto, discutem-se as vantagens de placas do sistema A0 , de titânio ou inox ${ }^{10}$.

\section{MÉTODOS}

Nos Serviços de Estomatologia e Cirurgia de Cabeça e Pescoço do Complexo Hospitalar Heliópolis, Hosphel, São Paulo, de 1980 a 2000, foram diagnosticados 31 ameloblastomas da mandíbula, e destes, 22 foram submetidos à cirurgia conservadora ou não. $\mathrm{O}$ sexo mais atingido foi o feminino em $54,5 \%$ dos casos $(p=0,05)$, e a faixa etária para o tipo folicular (13 casos) foram as das $3^{\text {as }}$ e 4 as $^{\text {a }}$ décadas, e para os tipos plexiforme (seis casos), e unicísticos (três casos) $(\mathrm{p}<0,9)$, foi a 3a década. Quanto à raça, houve o predomínio da branca em $68 \%$ da casuística.

Quanto à localização do ameloblastoma de mandíbula, seis eram anteriores cruzando a linha média (cinco foliculares e um plexiforme), cinco de corpo (três plexiforme e dois unicísticos), seis de corpo e ângulo (quatro foliculares, um plexiforme e um unicístico) e cinco de corpo, ângulo, ramo vertical e côndilo (quatro foliculares e um plexiforme) (Figura 1).

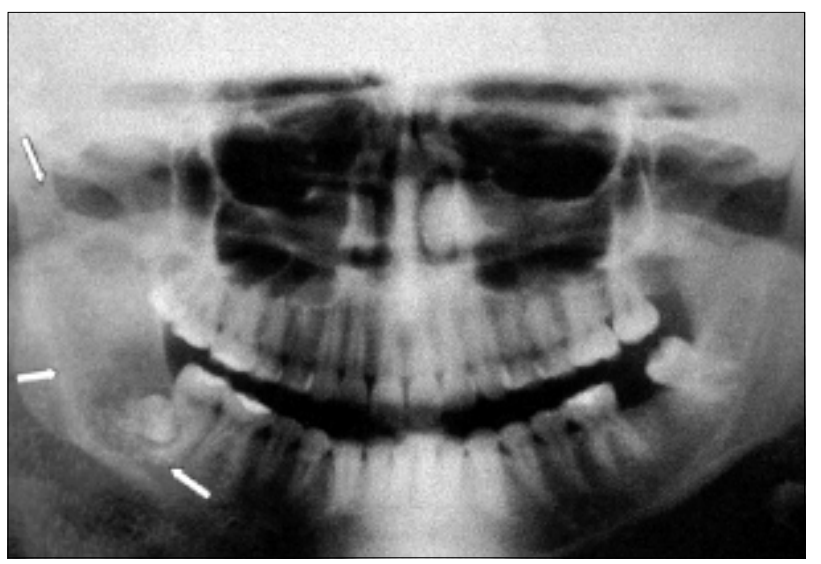

Figura 1. Ameloblastoma envolvendo corpo, ângulo, ramo e côndilo - radiografia panorâmica.

Quanto ao tratamento, este foi conservador em três casos onde foi feita enucleação ou curetagem (para a variedade unicística), sete ressecções marginais (três foliculares, quatro plexiformes) e 12 segmentares (dez foliculares e duas plexiformes).

No que diz respeito à reconstrução, esta foi indicada em sete pacientes (Tabela 1).

Para a utilização do enxerto (Tabela 2), foi utilizado o sistema A-0 de titânio com 2,4mm (em quatro pacientes com o tipo folicular) e de aço inox de $2,7 \mathrm{~mm}$ (em três pacientes, sendo dois foliculares e um plexiforme) ambas fixas com parafuso ao remanescente mandibular. Figuras 2 e 3.
Tabela 1

Tratamento cirúrgico não-conservador do ameloblastoma

\begin{tabular}{l|c|c|c|c}
\hline Reconstrução & $\begin{array}{c}\text { Tipo } \\
\text { Folicular }\end{array}$ & $\begin{array}{c}\text { Tipo } \\
\text { Plexiforme }\end{array}$ & $\begin{array}{c}\text { Tipo } \\
\text { Cístico }\end{array}$ & Total \\
\hline $\begin{array}{l}\text { Sem reconstrução } \\
\begin{array}{l}\text { Reconstrução com } \\
\text { crista ilíaca e } \\
\text { placa A-0 }\end{array}\end{array}$ & 7 & 5 & 3 & 15 \\
\hline Total & 6 & 1 & 0 & 7 \\
\hline
\end{tabular}

Tabela 2

Tipos histológicos versus fixação de enxerto de crista ilíaca

\begin{tabular}{l|c|c|c}
\hline Placas & $\begin{array}{c}\text { Tipo } \\
\text { Folicular }\end{array}$ & $\begin{array}{c}\text { Tipo } \\
\text { Plexiforme }\end{array}$ & Total \\
\hline Aço inox 2,7mm & 2 & 1 & 3 \\
Titânio 2,4mm & 4 & 0 & 4 \\
\hline Total & 6 & 1 & 7 \\
\hline
\end{tabular}

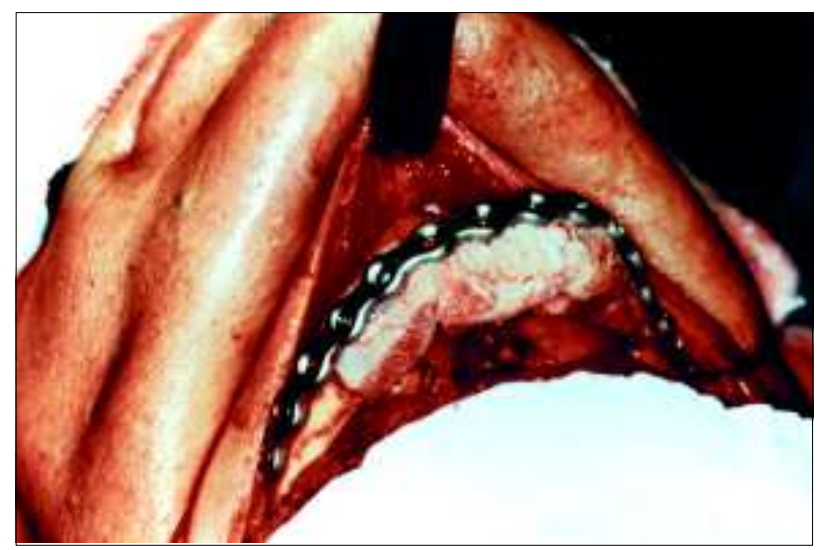

Figura 2. Enxerto de crista ilíaca não vascularizado. Fixação com placa do sistema A-0 de titânio $(2,4 \mathrm{~mm})$.

Para a análise estatística, foi utilizado o Teste de Hipótese para a média popoulacional com variância desconhecida de Latorre (1977).

\section{RESULTADOS}

O enxerto de crista ilíaca foi feito em sete dos 22 pacientes, sendo utilizadas as placas de estabilização do sistema A-0 (Tabela 2).

Quanto ao emprego das placas segundo à extensão da neoplasia, o critério de escolha foi feito considerando os cotos mandibulares remanescentes e as características texturais do osso onde a placa foi fixada. Dos sete casos, a 
exposição da placa foi a complicação mais comum, acompanhada de perda dos parafusos (Tabela 3).

\section{DISCUSSÃO}

A escolha do tratamento (conservador ou não) é em função do tamanho, tipo de lesão, localização e histopatologia ${ }^{11}$. Para a conduta radical, a mandibulectomia segmentar deve incluir uma margem cirúrgica óssea de 1 a $2 \mathrm{cms}$ na mandíbula, sem prejuízo da radicalidade, com inclusão de todas as porções da mandíbula até a desarticulação ${ }^{12}$, sem todavia considerarmos que a não reconstrução leva os pacientes para deformidades estéticas e distúrbios funcionais da mastigação, deglutição e fonação ${ }^{13}$.

As ressecções segmentares implicam a fixação obrigatória do enxerto ósseo aos cotos remanescentes da mandíbula, visto que a mesma se movimenta por ação da musculatura mastigatória. Assim, o bloqueio intermaxilar, para evitar rotação dos côndilos, que se compõe com barras ou amarrias, e com miniplacas e parafusos para pacientes dentados e para pacientes edêntulos, goteiras acrílicas confeccionados no pré-operatório, como fizemos em nossos pacientes, é necessário no transoperatório. O critério fundamental para o uso de enxerto autógeno não vascularizado como o que usamos em sete casos foi a presença de um leito de tecido mole para revascularizar a reconstrução, e a possibidade de cobertura do enxerto com retalhos de proteção ${ }^{14}$.

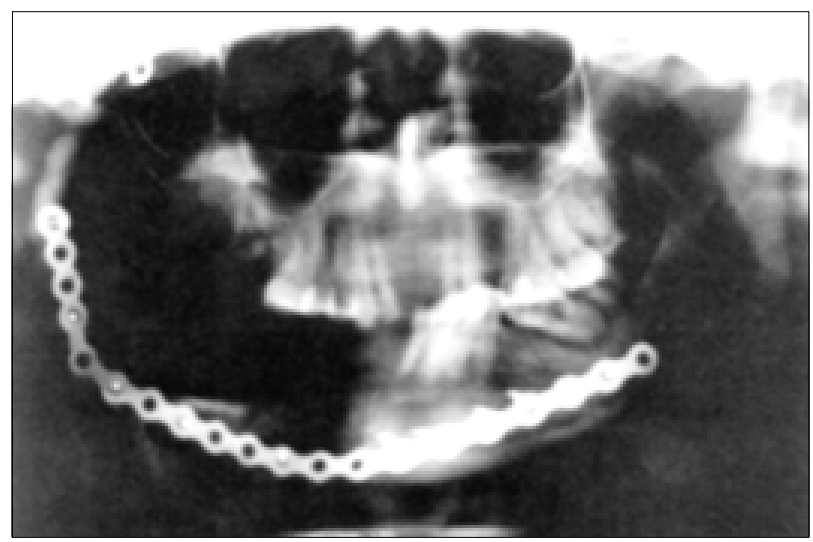

Figura 3. Ameloblastoma folicular de corpo, ângulo, ramo e côndilo; reconstruído com enxerto de crista ilíaca não vascularizado fixo com placa do sistema A-0 de titânio (2,4mm) - radiografia panorâmica.
Tabela 3

Complicações do emprego de placa A-0

\begin{tabular}{l|c}
\hline Placa & $\begin{array}{c}\text { Exposição da Placa } \\
\text { com Perda de Parafuso }\end{array}$ \\
\hline A/0 de inox $2,7 \mathrm{~mm}$ & 2 \\
A/0 de titânio $2,4 \mathrm{~mm}$ & 1 \\
\hline Total & 3 \\
\hline
\end{tabular}

Em nossa casuística de 22 ameloblastomas, seis do tipo folicular e um plexiforme receberam enxerto não vascularizado de crista ilíaca, escolha feita ao considerarmos a possibilidade de estabilizar uma espessura óssea equivalente à perda mandibular, o que se torna problemático ao considerarmos as outras opções como a fíbula, o rádio e a costela. Entretanto, deve-se levar em conta que este tipo de enxerto tem uma reabsorção mínima de $50 \%$, o que requer cuidados operatórios imediatos de cinco a seis semanas ${ }^{15}$.

Para a fixação dos enxertos, utilizamos placas de reconstrução do sistema A-0 de titânio ou aço inox, utilizando quatro parafusos em cada lado dos cotos ósseos, o que permitiu, em nossos pacientes, uma estabilidade adequada da placa, impedindo a sua fratura. Cabe lembrar que a placa de titânio que utiliza parafuso perfurado e oco (sistema Thorp), é vantajosa em relação à que utilizamos (sistema A-0), visto que a osteointegração se faz melhor quando utilizamos o primeiro, mas não é disponível para uso rotineiro entre nós ${ }^{16}$.

Quanto às complicações (três em nossos sete casos), a exposição da placa independe do material utilizado, enquanto que a fratura e a perda de parafusos são mais comuns no sistema A-0 por nós empregado em relação ao Thorp referido na literatura ${ }^{9,15}$ e 16 . Quando há exposição extra-oral, a perda de parafusos é concomitante e a retirada da placa é imediata, fato ocorrido em duas de nossas três complicações. No $3^{\circ}$ caso, onde evidenciou-se a exposição intra-oral, a placa foi removida somente três anos depois. Nesta situação, a tentativa de recolhimento da placa é desejável através de retalhos intra-orais, apesar da exposição reincidir usualmente como ocorreu em nosso paciente.

Em síntese, o tratamento cirúrgico dos ameloblastomas através de reconstrução com enxerto autólogo de crista ilíaca deve ser tentado, considerando os baixos índices de complicação em nossa experiência com os não vascularizados, bem como nos microcirúrgicos relatados na literatura.

\begin{abstract}
Background: To determine of the real validity of non vascularized iliac crest graft in the surgical treatment of ameloblastoma of the mandible. Methods: From 1980 to 2000, at the Head and Neck and Oral Semiology Services of Heliópolis Hospital, 29 patients were submitted to differents approaches, from which 7 received a nonvascularized autologus iliac crest graft fixed with A-0 titanium (4 cases) or inox (3 cases) systems plates for folicular (6 cases) and plexiform (1 case) ameloblastomas. Results: We used a test of hypothesis for the population
\end{abstract}


mean with unknown in variance. There was plate exposition in 3 cases (40\%), when the tumor overpass midline, and complete sucess in 4 cases (60\%) with no complications of this reconstructive approach for mandible ameloblastoma. Conclusions: In spite of the incidence of plate expesure, this technique is indicated for mandible reconstruction in patients with amelablastoma.

Key Words: Ameloblastoma; Iliac crest; Reconstruction; Plates.

\section{REFERÊNCIAS}

1. Ivey RH, Churchill HR. The need of a standardized surgical and pathological classification of tumors and anomalies of dental origin. Am Assoc Dent Sch Trans 1930; 7:240-5.

2. Kramer IRH. The World Health Organisation: Histological Typing of Odontogenic Tumors: An Introduction to the Second Edition. J Dent Assoc South Afr 1992; 47:208-10

3. Regezi JA, Kerr DA, Courtney RM. Odontogenic Tumors: Analysis of 706 Cases. J Oral Surg 1978; 36:771-8.

4. Philipsen HP, Ormiston IW, Reichart PA. The Desmo- and Osteoplastic Ameloblastoma. Histologic Variant or Clinicopathologic Entity? Case Report. Int J Oral Maxillofac Surg, 1992; 21:352-7.

5. Reichart PA, Philipsen HP, Sonner S. Ameloblastoma: Biological Profile of 3677 Cases. Eur J Cancer 1995; 31B:86-99.

6. Martins RH, Andrade Sobrinho J, Rapoport A, Rosas MP Histopathologic features and management of ameloblastoma: study of 20 cases .São Paulo Med J 1999; 117:171-4.

7. Gardner DG, Corio RL. The Relationship of Plexiform Unicystic Ameloblastoma to Conventional Ameloblastoma. Oral Surg Oral Med Oral Pathol 1983; 56:54-60.

8. David DJ, Tan E, Katsaros J, Sheen R. Mandibular Reconstruction with Vascularized Iliac Crest: A 10 - Year Experience. Plast Reconstr Surg 1988;82:792-803.

9. Blackwell KE, Buchbinder D, Urken ML. Lateral Mandibular Reconstruction Using Soft- Tissue Free Flaps and Plates. Arch Otolaryngol Head Neck Surg 1996; 122:672-8.

10. Schöning H, Emshoff R. Primary Temporary AO Plate Reconstruction of the Mandible. Oral Surg Oral Med Oral Pathol 1998; 86:667-72.
11. Vasan NT. Recurrent Ameloblastoma in an Autogenous Bone Graft After 28 Years: a Case Report. N Z Dent J 1995; 91:12-3.

12. Feinberg SE, Steinberg B. Surgical Management of Ameloblastoma. Current Status of the Literature. Oral Surg Oral Med Oral Pathol Oral Maxillofac Surg 1996;81:383-8.

13. Carlson ER, Marx RE. Mandibular Reconstruction Using Cancellous Cellular Bone Grafts. J Oral Maxillofac Surg 1996; 54:889-97.

14. Wells MD. Mandibular Reconstruction Using Vascularized Bone Grafts. J Oral Maxillofac Surg 1996; 54:883-8.

15. Futran ND, Urken ML, Buchbinder D, Moscoso JF, Biller HF. Rigid Fixation of Vascularized Bone Grafts in Mandibular Reconstruction. Arch Otolaryngol Head Neck Surg 1995; 121:70-6.

16. Vuillemin T, Raveh J, Sutter F. Mandibular Reconstruction with the Titanium Hollow Screw Reconstruction Plate (THORP) System: Evaluation of 62 Cases. Plast Reconstr Surg 1988; 82:804-14.

Endereço para correspondência:

Prof. Dr. Abrão Rapoport

Praça Amadeu Amaral, 47 - cj. 82

01327-010 - São Paulo - SP 